\title{
Numerical investigation of deposition and dispersion of aerosols in a concentric annulus
}

\author{
Milad Gerailinejad, Davood Domairy Ganj, Mehran Khaki \\ Department of Mechanical Engineering, Islamic Azad University, Sari Branch, Sari, Iran \\ Received: 1 February 2016, Accepted: 16 February 2016 \\ Published online: 5 May 2017.
}

\begin{abstract}
In the present analysis, we studied numerically the effect of natural convection on deposition and dispersion of aerosols in a concentric horizontal annulus by means of control volume based finite element method. The governing equation is assumed for laminar, incompressible and 2D fluid flow while a Lagrangian description was used for simulation of spherical, solid particles immersed in the flow. The inner cylinder was under a constant heat flux and the outer one was maintained at a constant temperature $\left(T_{c}\right)$. The Drag, Lift, Brownian and gravity forces were considered in the equation of particle motion. The results showed that Rayleigh number and increment of the aspect ratio change the rate of deposition as showed in the figures.
\end{abstract}

Keywords: Control volume based finite element method (CVFEM), concentric annulus, aerosol, deposition and dispersion.

\section{Introduction}

The subject of particle and the issues surrounded it such as deposition, dispersion and aggregation is one of the most interesting topics for researchers to investigate and simulate its physical unpredictable motion. As known we can see the existence of aerosols in air pollution, human health, medical cure, soiling of historical monuments, and determination of indoor air quality and many other applications that show the importance of research in this field.

A numerical approach for transport of suspended small particles was developed by Li and Ahmadi [1, 2]. Akbar et al. [3] studied the behavior of sub-micron particles in a laminar free convection in a square enclosure. Thatcher et al. [4] investigated particle deposition mechanism in a closed cavity experimentally. Their cavity had two cold walls (the top and the left walls) and two heated walls (the bottom and the right walls). Heim et al. [5] studied the particle losses to the inside walls of a T-shaped micro-reactor as a function of operating conditions. Numerical investigation was performed by Golkarfard et al. [6] to study the transport and deposition of aerosol particles in laminar mixed-convection flow in a lid-driven cavity with two heated obstacles. They studied the effects of thermophoresis and free convection on deposition of particles on the obstacles. H. Hassanzadehet al. [7] investigated numerically dispersion and removal of microaerosol particles in a horizontal concentric annulus by Lattice Boltzmann Method and Lagrangian Runge-Kutta procedure with the assumption of one-way coupling. G. H. Bagheri et al. [8] studied transport and deposition of solid particles in a differentially heated cavity at high Rayleigh numbers up to $10^{8}$ using an Eulerian-Lagrangian computational method. T. H. Kuehn, R. J. Goldstein [9] investigated an experimental and theoretical study of natural convection in the annulus between horizontal concentric cylinders. M. Sheikholeslami et al. [10] studied the effect of a magnetic field on natural convection in a half-annulus enclosure with one wall under constant heat flux using control volume based finite element method. S.M. Seyyedi et al. [11] simulated natural convection heat transfer of $\mathrm{Cu}$-water nanofluid in an annulus enclosure using Control Volume based Finite Element Method (CVFEM). And some other researches based on this method was conducted by M. Sheikholeslami et al. [12, 13]. 
In the present study we have studied particle deposition and dispersion in a horizontal annulus with natural convection. We showed the influence of aspect ratio and Rayleigh number on the rate of deposition. We considered 1000 solid particles that have the same physical properties with diameter of $d_{p}=10 \mu \mathrm{m}$.

\section{Geometry and boundary conditions}

As shown in Fig.1 the inner cylinder includes a constant heat flux while the outer one is maintained at a constant temperature $\left(\mathrm{T}_{c}\right)$. The space between cylinders is filled with air and the initial position of aerosols considered randomly.

The governing equation of 2D, laminar and incompressible natural convection fluid flow is assumed for this problem.

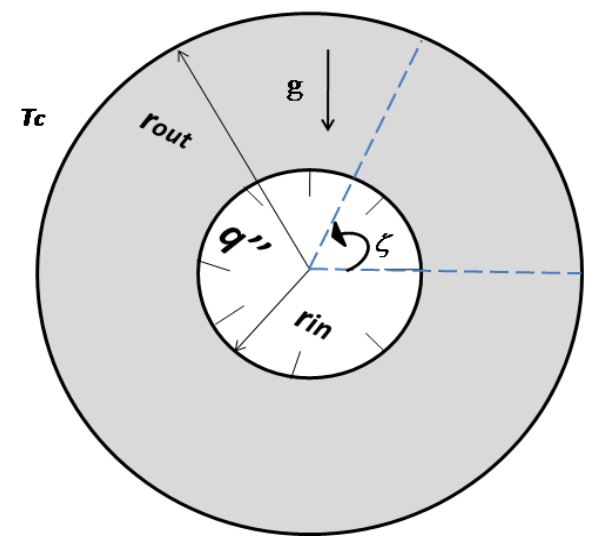

Fig. 1: Schematic of geometry and boundary conditions.

The continuity, Navier-Stokes and energy Equations are as follow respectively:

$$
\begin{aligned}
& \frac{\partial u}{\partial x}+\frac{\partial v}{\partial y}=0 \\
& u \frac{\partial u}{\partial x}+v \frac{\partial u}{\partial y}=-\frac{1}{\rho} \frac{\partial p}{\partial x}+v\left(\frac{\partial^{2} u}{\partial x^{2}}+\frac{\partial^{2} u}{\partial y^{2}}\right) \\
& u \frac{\partial v}{\partial x}+v \frac{\partial v}{\partial y}=-\frac{1}{\rho} \frac{\partial p}{\partial y}+v\left(\frac{\partial^{2} v}{\partial x^{2}}+\frac{\partial^{2} v}{\partial y^{2}}\right)+\beta g\left(T-T_{c}\right) \\
& u \frac{\partial T}{\partial x}+v \frac{\partial T}{\partial y}=\alpha\left(\frac{\partial^{2} T}{\partial x^{2}}+\frac{\partial^{2} T}{\partial y^{2}}\right)
\end{aligned}
$$

Stream function and vorticity are defined below.

$$
u=\frac{\partial \psi}{\partial y}, v=-\frac{\partial \psi}{\partial x}, \omega=\frac{\partial v}{\partial x}-\frac{\partial u}{\partial y}
$$

The stream function satisfies the continuity Eq. (1). The vorticity equation is obtained by eliminating the pressure between the two momentum equations, i.e. by taking y-derivative of Eq. (2) and subtracting it from the x-derivative of Eq. (3). Non- 
dimensional variables are

$$
\begin{aligned}
& X=\frac{x}{L}, Y=\frac{y}{L}, \Omega=\frac{\omega L^{2}}{\alpha}, \Psi=\frac{\psi}{\alpha} \\
& \Theta=\frac{T-T_{c}}{\Delta T}, \Delta T=\frac{q^{\prime \prime} L}{k}
\end{aligned}
$$

where $\Theta, \Psi, \Omega, Y, X$ are dimensionless temperature, stream function, vorticity and space coordinates respectively.

Also $R a=g \beta L^{3} q^{\prime \prime} L /\left(\alpha v k_{f}\right)$ Rayleigh and $\operatorname{Pr}=v / \alpha$ Prandtl number are defined. And the local Nusselt number for hot wall is defined as

$$
N u_{\text {local }}=\frac{1}{\Theta(\zeta)}
$$

Here we introduce some important dimensionless numbers known as Knudsen, Mach, Reynolds and stokes number.

$$
\begin{aligned}
K n^{p} & =\frac{2 \lambda}{d} \\
M a^{p} & =\frac{\left|U^{f}-U^{p}\right|}{C^{f}} \\
\operatorname{Re}^{P} & =\frac{\left|U^{f}-U^{p}\right| d}{v} \\
S t k & =\frac{\tau^{p}}{\tau^{f}} .
\end{aligned}
$$

Eq. (12) describes particle equation of motion in Lagrangian coordinate system.

$$
\begin{aligned}
\frac{d v_{p}}{d t} & =\sum F_{i} \\
\frac{d u_{p, i}}{d t} & =\frac{1}{\tau}\left(u_{i}-u_{p, i}\right)+g_{i}\left(1-\frac{1}{S}\right)+F_{L, i}+n_{i}(t) \\
\frac{d x_{i}}{d t} & =u_{p, i},
\end{aligned}
$$

and the first term at right hand of the equation is drag, the second term is gravity, the third term is Lift and the last one denotes Brownian force.

By introducing Eq. (13) $\tau_{p}$ as the relaxation time and replacing it in Eq. (12) we will have new form of particle motion equation as Eq. (17).

$$
\tau_{p}=\frac{m_{p} C_{C}}{3 \pi \mu d}=\frac{d^{2} \rho_{p} C_{C}}{18 v}
$$

As an example in the reference [8] the relationship and formula for $\mathrm{C}_{c}, \mathrm{~F}_{D}, \mathrm{~F}_{B}$ and etc are described as below. Cunningham'sfactor is

$$
C_{C}=1+\frac{2 \lambda}{d}\left(1.257+0.4 \exp \left(\frac{-1.1 d}{2 \lambda}\right)\right)
$$


The drag force is defined as

$$
F_{D}=\frac{1}{2} C_{D} \rho U^{2} A
$$

and the Brownian force $n_{i}(t)$ is

$$
\begin{aligned}
n_{i}(t) & =G_{i} \sqrt{\frac{\pi S_{n n}}{\Delta t}} \\
S_{n n} & =\frac{2 k_{b} T \beta}{\pi m_{P}} \\
G_{1} & =\sqrt{-2 L n U_{1}} \cos 2 \pi U_{2}, G_{2}=\sqrt{-2 \operatorname{Ln} U_{1}} \sin 2 \pi U_{2} .
\end{aligned}
$$

$G_{i}$ is zero-mean, unit variance Gaussian random number selected at each time step and $K_{b}$ is the Boltzmann constant $\left(k_{b}=1.38 \times 10^{-23} \mathrm{~J} / K\right)$.

$$
\frac{d V_{p}^{i}}{d t}=\frac{1}{\tau_{p}}\left(V_{g}^{i}-V_{p}^{i}\right)+\frac{F_{L}^{i}}{m^{p}}+F_{B}^{i}+g^{i}
$$

Now we introduce the physical properties of studied particle (silicon dioxide) in the table 1.

Table 1: physical properties of silicon dioxide.

\begin{tabular}{|ccc|}
\hline $\begin{array}{c}\rho \\
(\mathbf{k g} / \mathbf{m} 3)\end{array}$ & $\begin{array}{c}\mathbf{C}_{p} \\
(\mathbf{J} / \mathbf{k g ~ k})\end{array}$ & $\begin{array}{c}\mathbf{K} \\
(\mathbf{W} / \mathbf{m k})\end{array}$ \\
\hline 2220 & 745 & 1.38 \\
\hline
\end{tabular}

\section{Validity}

In this study we compared the results with some previous reliable researches. Here we show isotherm contours of the present study and the one by T. H. KUEHN [9] and also the equivalent thermal conductivity on inner cylinder in Figs. 2,3.
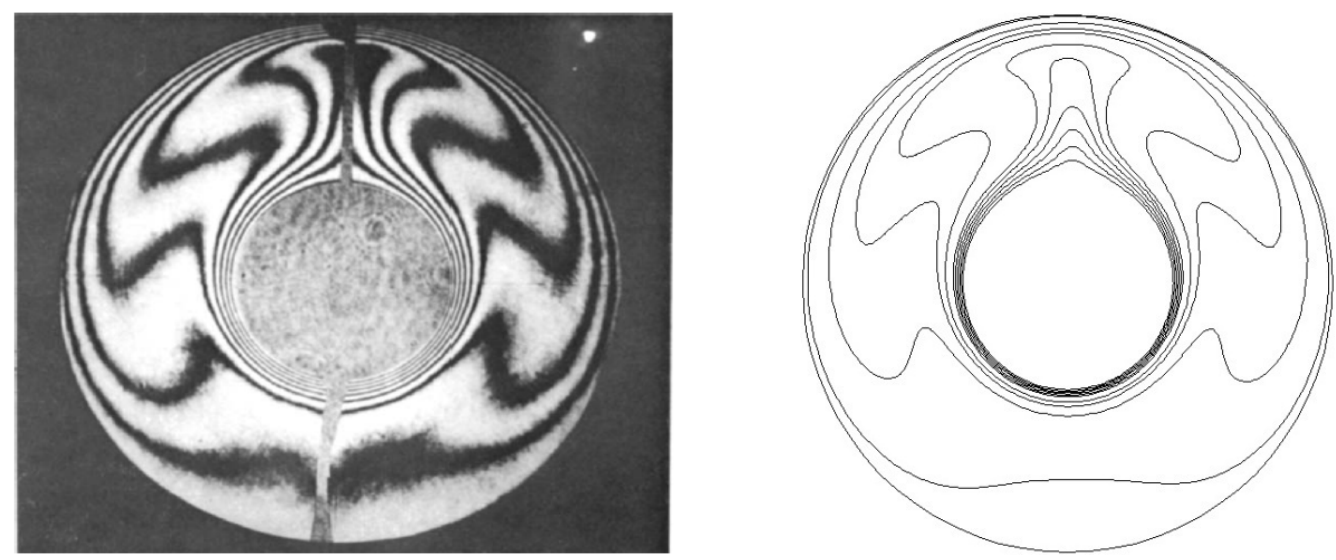

Fig. 2: Comparison of isotherms, present study (right) and Kuehn [9] (left). 


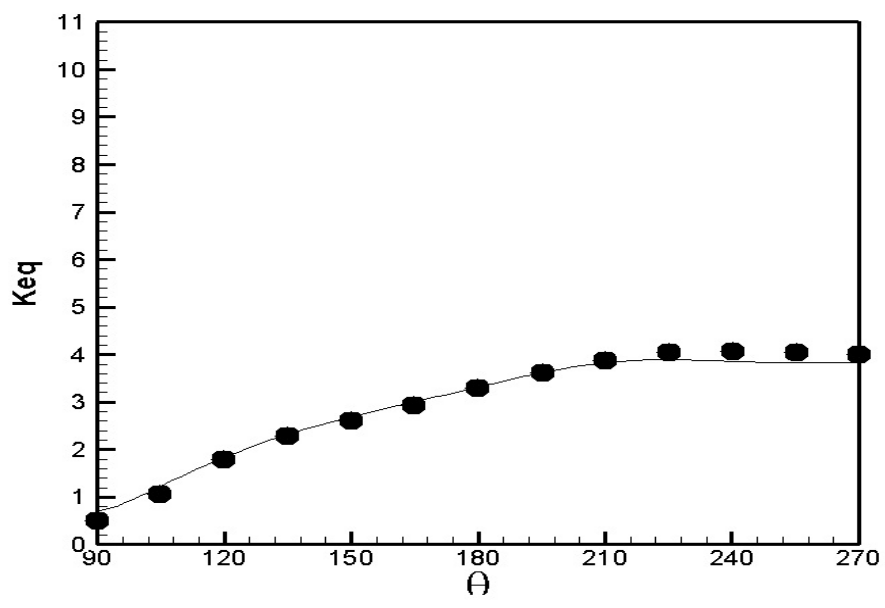

Fig. 3: Comparison of equivalent conductivity on inner cylinder, present study (filled circle), Kuhen (solid line).

A parameter has been defined in several past researches known as deposition fraction or removal fraction $(R F)$ that shows the number of particles deposited on the both cylinders in comparison with the whole particles injected to the geometry just like as Eq. (18)

$$
R F=\operatorname{fracN}_{d} N_{t},
$$

where $N_{d}$ denotes number of deposited particle and $N_{t}$ shows the number of all particle injected between cylinders. So Fig. 4 presented the validity of our study by comparison two cases as mentioned here.

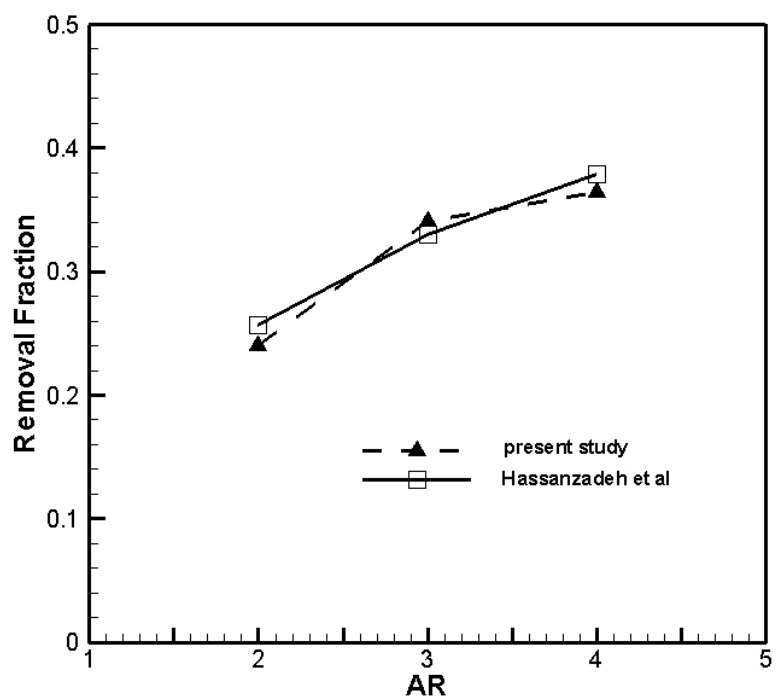

Fig. 4: Comparison of removal fraction of particles between present study and Hassanzadeh et al. [7].

As mentioned in Figs. 2, 3 and 4 acceptable agreements were found between results. We tried several mesh sizing to achieve grid independent solutions and we found that $61 * 181$ mesh size is appropriate for this case. 


\section{Results and discussion}

In the present study we have studied particle deposition and dispersion in a horizontal annulus with natural convection. We investigated the influence of aspect ratio and Rayleigh number on the particle motion. We presented results for different AR, 2, 3, 4 and 5 and different Rayleigh numbers $10^{3}, 10^{4}$ and $10^{5}$.
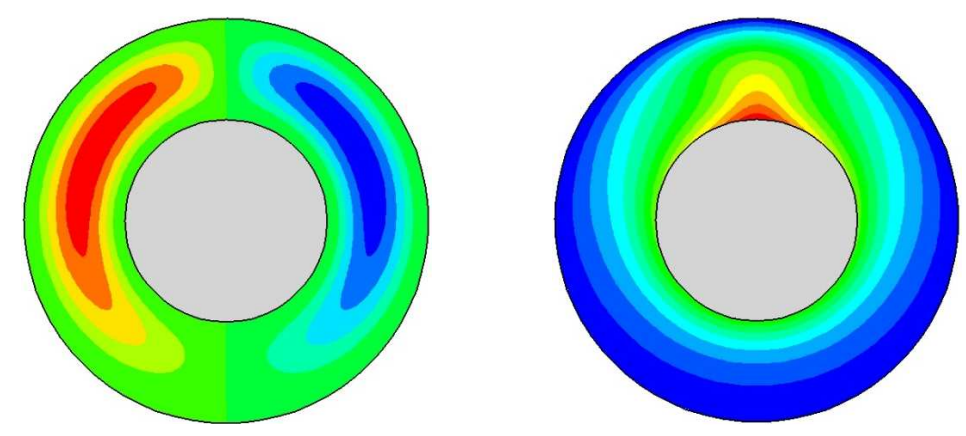

$\mathrm{AR}=2$
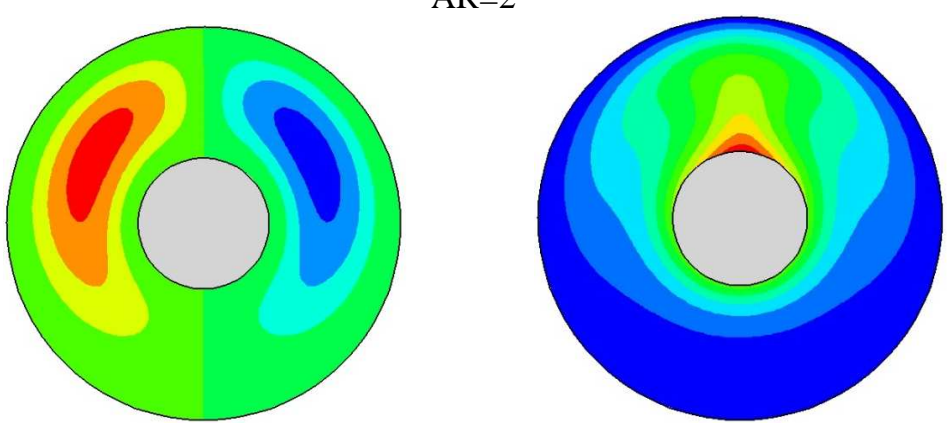

$\mathrm{AR}=3$
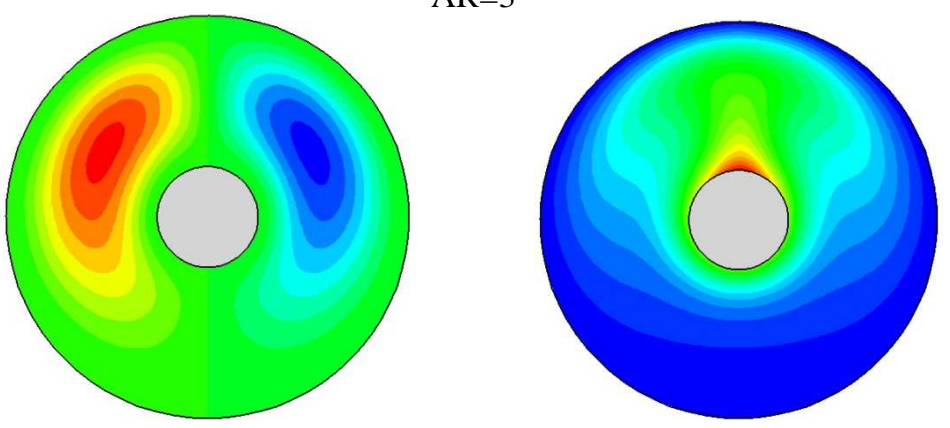

$\mathrm{AR}=4$
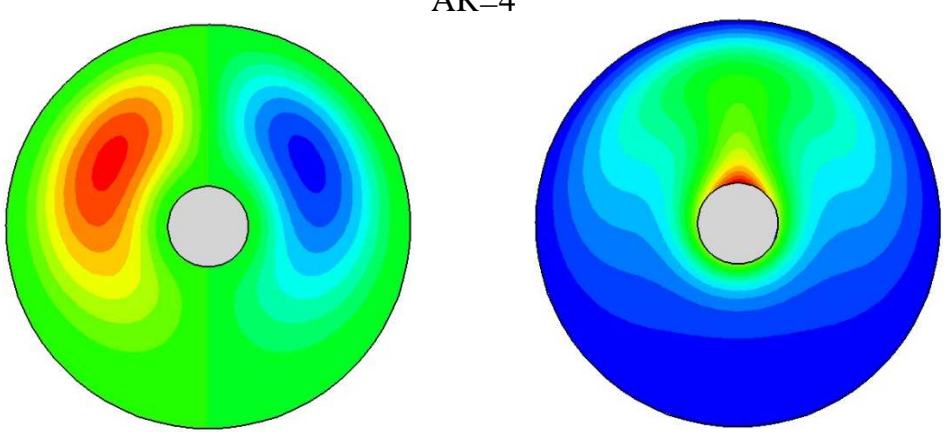

$\mathrm{AR}=5$

Fig. 5: Comparison of isotherms (left) and streamlines (right) contours at $\mathrm{Ra}=104$ for different AR. 

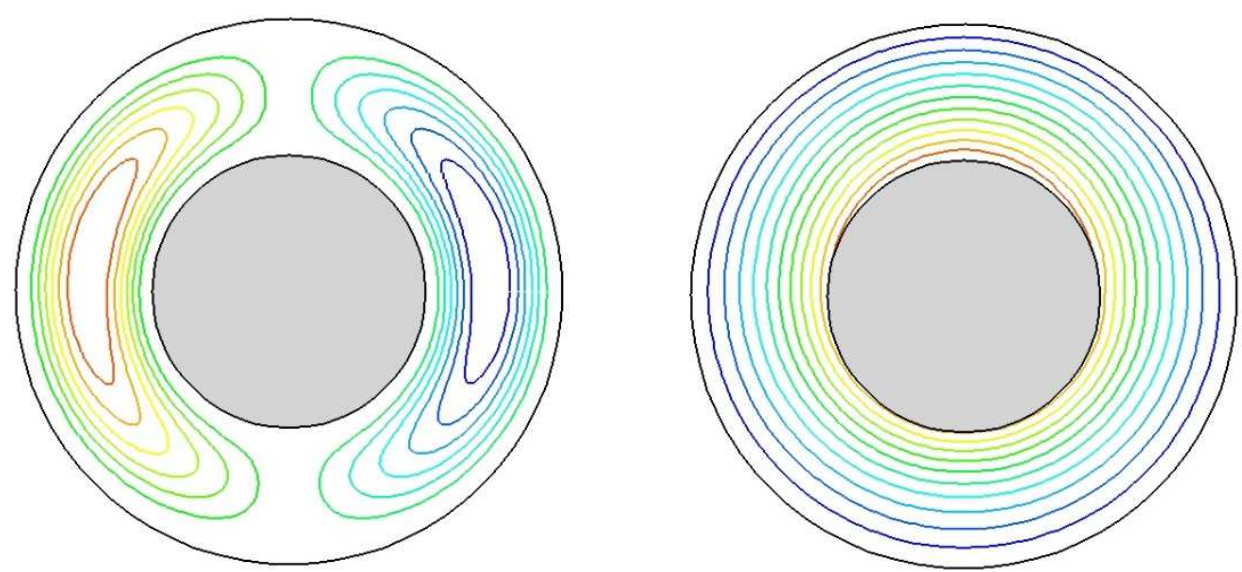

$R a=10^{3}$
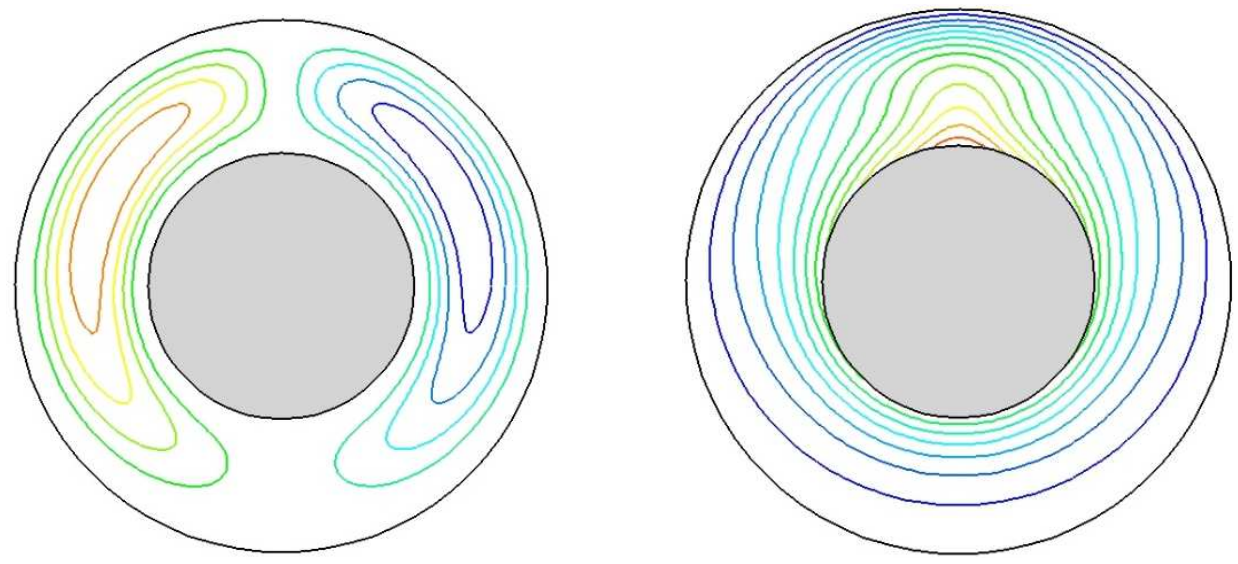

$R a=10^{4}$
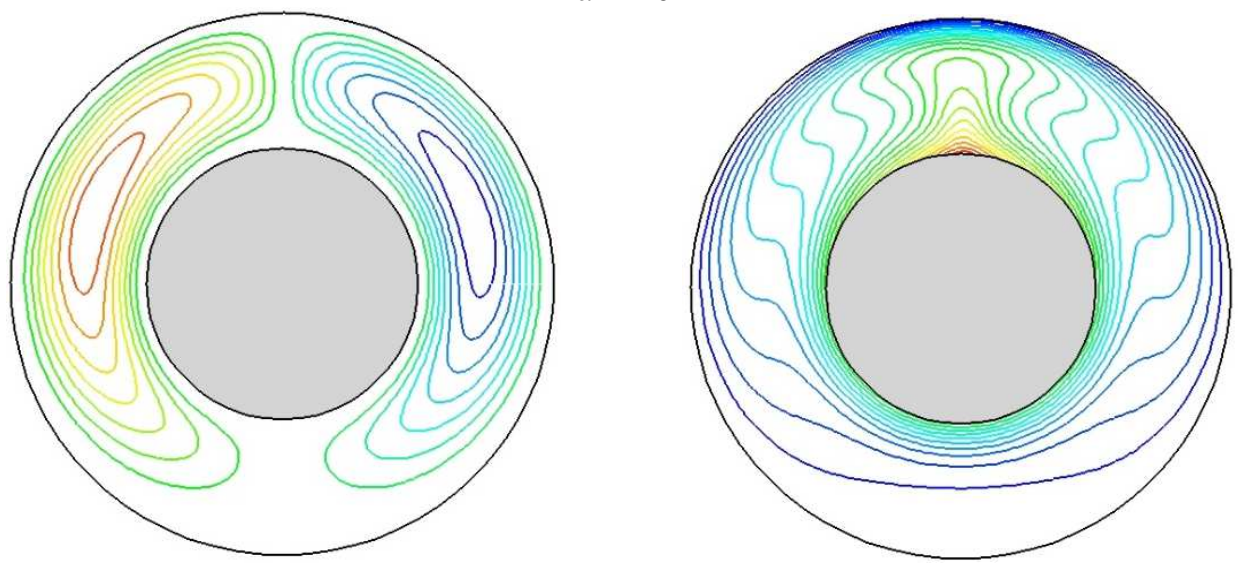

$R a=10^{5}$

Fig. 6: Comparison of isotherms (left) and streamlines (right) contours at $A R=2$ for different Ra number.

Fig.4 indicates the pattern of isotherm changes by increment of aspect ratio. The streamline contours also show the strength of the flow field that has inverse relation with AR. Fig. 5 shows that as the Rayleigh number increases up to $R a=10^{5}$, the isotherms become more distorted due to the domination of convective heat transfer. In addition, the thermal boundary layer on the surface of the inner cylinder becomes thinner at higher Rayleigh number. Fig. 6 presented the position of aerosols at $t=50 \mathrm{~s}$ and $R a=10^{3}$ for different AR. Fig. 7 shows the trend of local Nusselt number changes on the hot cylinder for different aspect ratio at $R a=10^{4}$. As you can see we have increase in local Nusselt number by raising AR. 


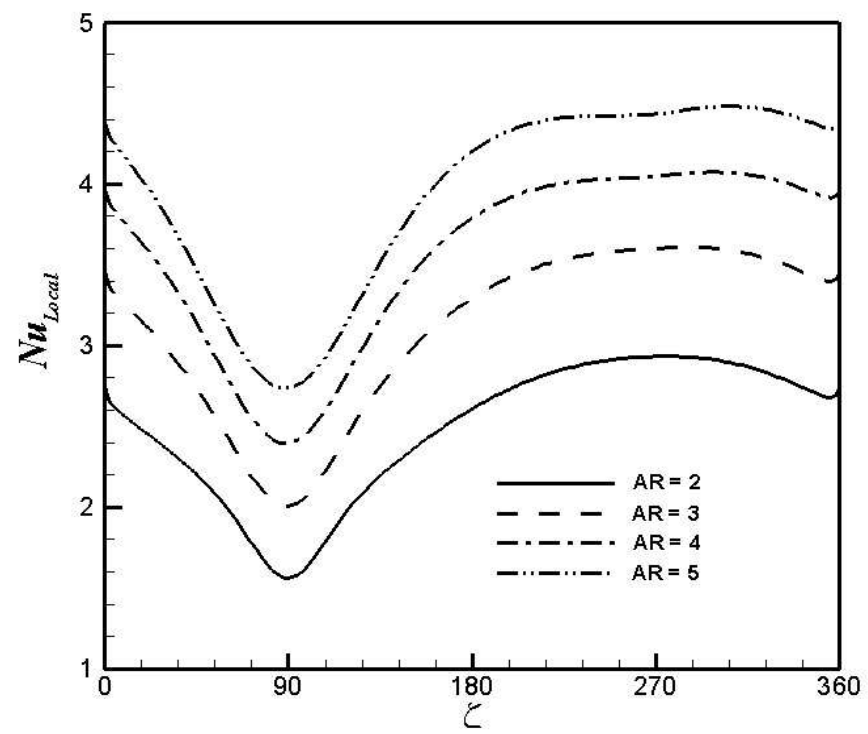

Fig. 7: Presentation of local Nusselt number on the hot cylinder for different $\mathrm{AR}$ at $R a=10^{4}$.
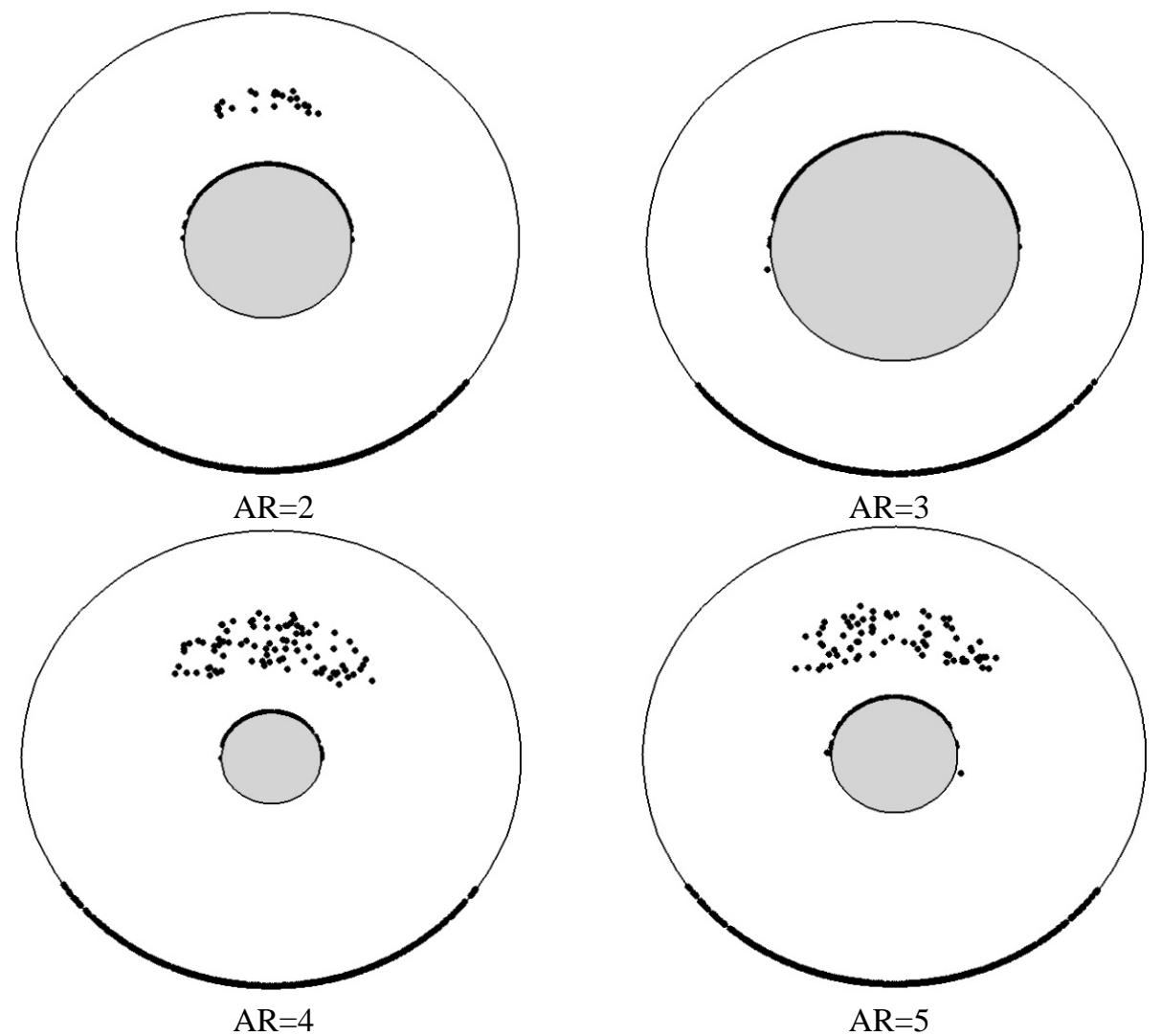

Fig. 8: Instantaneous snapshots of particle locations at $t=50 \mathrm{~s}$ at $R a=10^{3}$. 

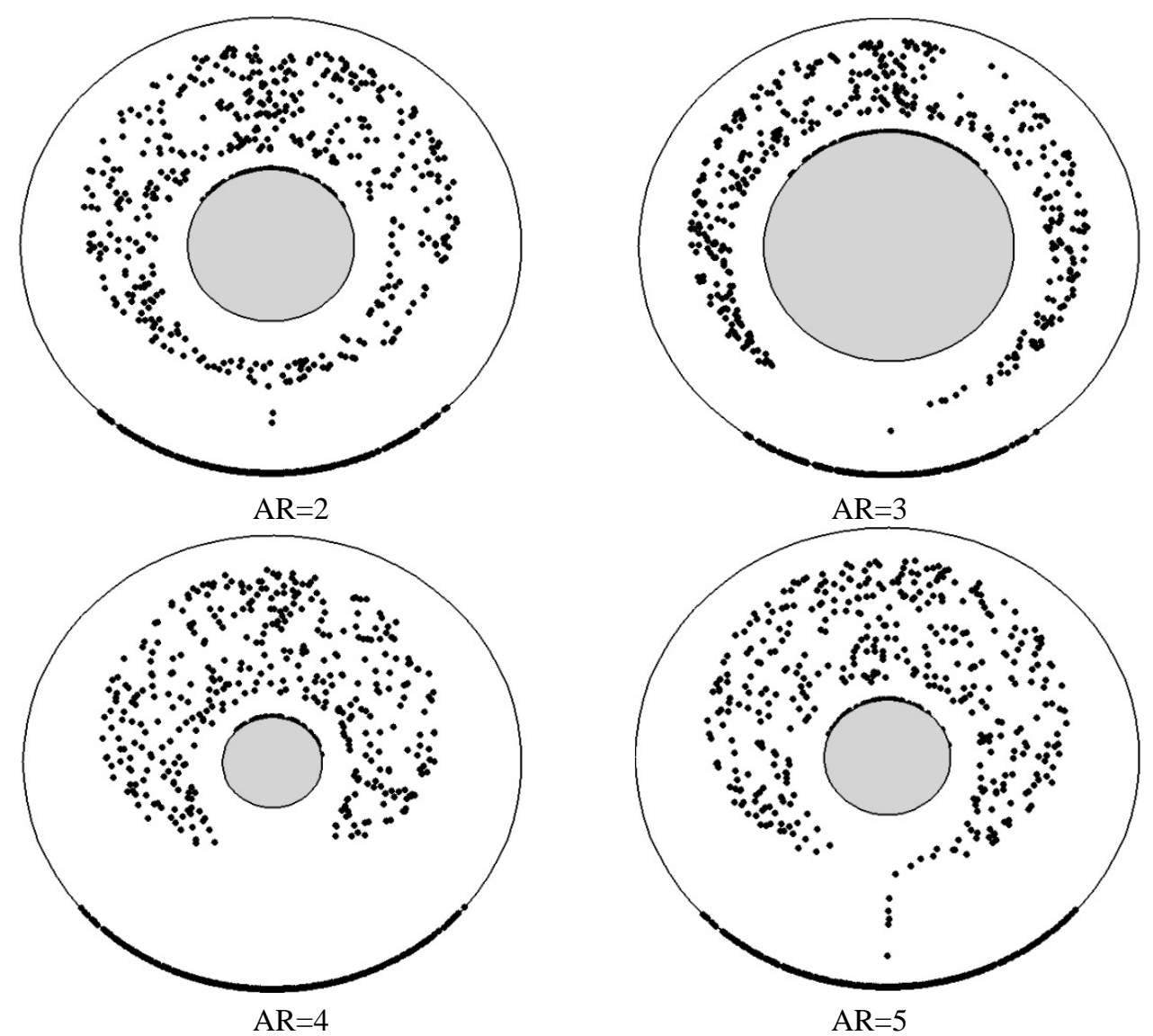

Fig. 9: Instantaneous snapshots of particle locations at $t=50 \mathrm{~s}$ at $R a=10^{4}$.

Table 2: Deposition fraction for different AR in presented Ra numbers at $t=50 \mathrm{~s}$.

\begin{tabular}{|ccccc|}
\hline & $\mathrm{AR}=2$ & $\mathrm{AR}=3$ & $\mathrm{AR}=4$ & $\mathrm{AR}=5$ \\
\hline$R a=10^{3}$ & 0.999 & 0.977 & 0.921 & 0.911 \\
\hline$R a=10^{4}$ & 0.586 & 0.564 & 0.596 & 0.615 \\
\hline$R a=10^{5}$ & 0.284 & 0.378 & 0.422 & 0.474 \\
\hline
\end{tabular}

At $R a=10^{3}$ as you can see for $A R=2$, almost the whole aerosols deposit on the both cylinders. By raising aspect ratio we can find more dispersed particles between hot and cold walls due to increasing of flow field strength. Due to the high density of aerosols in this case, and the weak flow field at low Rayleigh number on the other hand, we see that in less aspect ratio deposition happens aggressively because of short distance between cylinders. Fig.7 shows that deposition increases by enhancing aspect ratio except for $\mathrm{AR}=3$. The reason is that deposited particles on the hot wall reduce greatly and remain dispersed between cylinders as gravity and buoyancy forces became balanced. With consideration of Figs 7-9 and table 1 we can see that in a constant AR, the deposition fraction decreases by enhancing Ra number. We presented results for $d_{p}=10 \mu \mathrm{m}$ and we choose relaxation time of the particle as the time-step of this study.

\section{Conclusion}

In this case we have studied natural convection in a concentric annulus with outer cold wall and inner heated wall. We injected 1000 particles in laminar, incompressible and steady-state fluid flow and followed the trajectories of the aerosols 

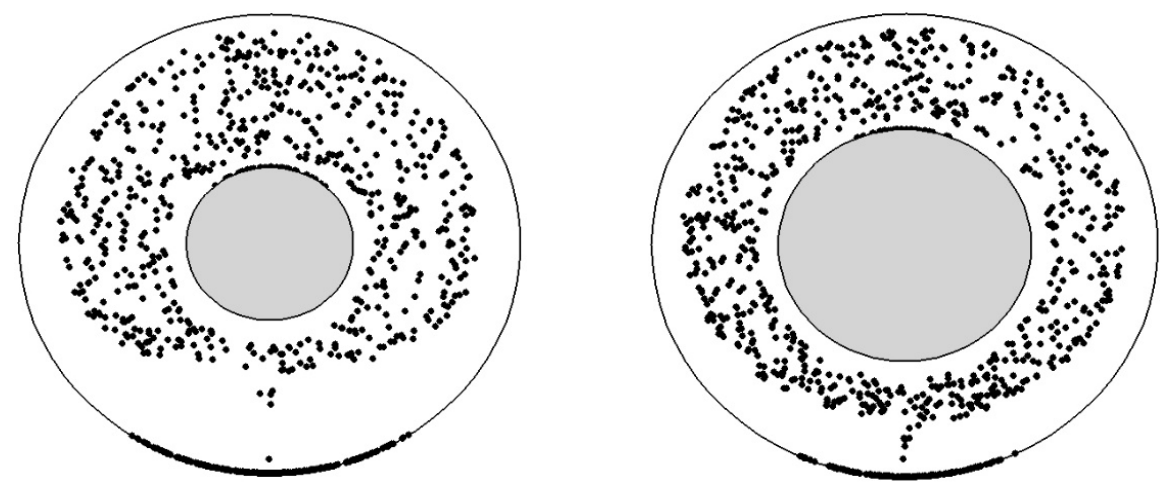

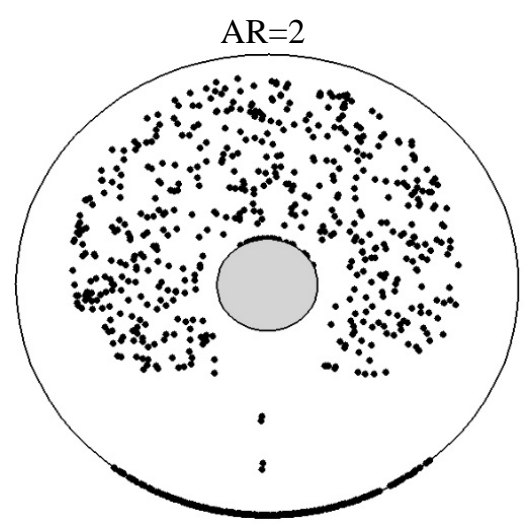

$\mathrm{AR}=4$

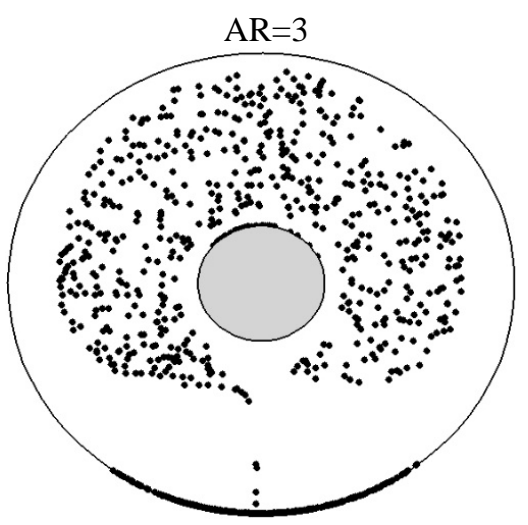

$\mathrm{AR}=5$

Fig. 10: Instantaneous snapshots of particle locations at $t=50 \mathrm{~s}$ at $R a=10^{5}$.

to analyze the pattern of deposition and dispersion. As mentioned in figures we concluded that by increment in aspect ratio the deposition increases for $R a=10^{4}, 10^{5}$ but we found opposite trend in $R a=10^{3}$ due to domination of gravity force over buoyancy. By increasing Rayleigh number the deposition decreased in all cases which means the aerosols tend to be dispersed in strong flow field.

\section{Competing interests}

The authors declare that they have no competing interests.

\section{Authors' contributions}

All authors have contributed to all parts of the article. All authors read and approved the final manuscript.

\section{References}

[1] Li, A. and Ahmadi, G., "Aerosol particle deposition with electrostatic attraction in a turbulent channel flow", Journal of Colloid and Interface Science, Vol. 158 (1993) 476-482.

[2] Li, A. and Ahmadi, G., "Deposition of aerosols on surfaces in a turbulent channel flow", International Journal of Engineering Science, Vol. 31 (1993) 435-451. 
[3] M.K. Akbar, M. Rahman, S.M. Ghiaasiaan, Particle transport in a small square enclosure in laminar natural convection, Journal of Aerosol Science 40 (2009) 747-761.

[4] Thatcher, T. L., Fairchild,W. A., andNazaroff,W.W. (1996). Particle Deposition from Natural Convection Enclosure Flow onto Smooth Surfaces. Aerosol Sci. Technol., 25:359-374.

[5] Heim, M.,Wengeler,R.,Nirschl,H.,\&Kasper,G.(2006).ParticledepositionfromaerosolflowinsideaT-shapedmicro-mixer. Journal ofMicromechanicsand Microengineering, 16, 70-76.

[6] V. Golkarfard, S.A. Gandjalikhan Nasab, A.B. Ansari, G.H. Bagheri, Numerical investigation on deposition of solid particles in a lid-driven square cavity with inner heated obstacles, Advanced Powder Technology, in press.

[7] Hassanzadeh Afrouzi, H., Farhadi, M., \& Abouei Mehrizi, A, .Numerical simulation of microparticles transport in a concentric annulus by Lattice Boltzmann Method. Advanced Powder Technology . 584-575. (2013).

[8] G. H. Bagheri, M. Salmanzadeh,V. Golkarfard, G. Ahmadi, Simulation of Solid Particles Behavior in a Heated Cavity at High Rayleigh Numbers, Aerosol Science and Technology, 46:1382-1391, 2012.

[9] T. H. KUEHN , R. J. GOLDSTEIN, an experimental and theoretical study of natural convection in the annulus between horizontal concentric cylinders, J . Fluid Mech. (1976), vol. 74, part 4 , pp. 695-719.

[10] M. Sheikholeslami, M. Gorji-Bandpy, D.D. Ganji ,Soheil Soleimani“ Effect of a magnetic field on natural convection in an inclined half-annulus enclosure filled with Cu-water nanofluid using CVFEM” Advanced Powder Technology 24 (2013) 980-991.

[11] S.M. Seyyedi, M. Dayyan, Soheil Soleimani, E. Ghasemi, Natural convection heat transfer under constant heat flux wall in a nanofluid filled annulus enclosure, Ain Shams Engineering Journal (2015) 6, 267-280.

[12] M. Sheikholeslamia, T. Hayatb, c, A. Alsaedic" MHD free convection of A12O3-water nanofluid considering thermal radiation: A numerical study" International Journal of Heat and Mass Transfer, 2016, Pages 513-52.

[13] M. Sheikholeslami, M.M. Rashidi, D.D. Ganji, Effect of non-uniform magnetic field on forced convection heat transfer of $\mathrm{Fe}_{3} \mathrm{O}_{4}-$ water nanofluid, Computer Methods in Applied Mechanics and Engineering, Volume 294, 1 September 2015, Pages 299-312. 